\title{
Forecasting of voltage disturbances at the beginning of ferroresonance phenomena in power systems using by a new approach of long-short term memory (LSTM): LSTM for Ferroresonance in power systems
}

\author{
Hidir Selcuk Nogay*
}

\section{DOI : 10.36909/jer.13467}

Department of Electrical and Energy, Kayseri University, Kayseri, 38280, Turkey.

*Email: nogay@kayseri.edu.tr; Corresponding Author.

\begin{abstract}
Ferroresonance is an unexpected, continuous, and undesirable event that causes excessive growth and distortion in the voltage and current waveform, whose cause is unknown and suddenly develops in power systems. To take precautions against the ferroresonance phenomenon, it is necessary to be able to predict how the distortions in the ferroresonance voltage will continue. Today, the ferroresonance problem has not been fully solved yet and more scientific studies are still needed. In this study, a new approach of an LSTM network has been developed that predicts the irregular and excessively large-amplitude continuing behavioral disturbances of the phase voltage in a real electrical power network exposed to ferroresonance. As a result of the study, the ferroresonance-voltage continuing in distorted waveform was estimated with an error of 0.0346 according to the Mean Absolute Percent Error (MAPE). The training data used in the study is only about $5 \%$ of all predicted ferroresonance voltage data. The successful estimation of $95 \%$ of the Ferreroresonance voltage using only about 5\%, proves the success of the LSTM model applied with a new approach for the study.
\end{abstract}

Key words: LSTM, ferroresonance phenomenon, identification, forecasting. 


\section{INTRODUCTION}

While electrical power systems operate normally under their usual loads, there may be shortterm sudden interruptions in the power system due to lightning strikes, commissioning of a backup transformer in the power system, or other similar reasons. These power outages sometimes cause fluctuating periodic distortions in the grid voltage that are inevitable and continue in a harmonic waveform. This phenomenon in power systems is called the ferroresonance phenomenon. The reason for the ferroresonance phenomenon may be inductances and capacitances in the network as well as switching errors or natural phenomena such as lightning. Therefore, as it is not known exactly what causes the ferroresonance event, it is almost impossible to detect it in advance or to detect it instantly. These overcurrent and overvoltage spikes and high-level harmonics caused by the ferroresonance phenomenon cannot be suppressed by conventional suppression methods. Current and voltage distortions caused by ferroresonance in power systems can cause serious malfunctions in the network (Tugai, 2015, Yang et al., 2017a, Boutora \& Bentarzi, 2018). To reach solutions for protection and delay from over current and voltage caused by ferroresonance, it is necessary to recognize the ferroresonance phenomenon quickly (Rezaei, 2017). In a study, the smart solid-state stress suppressor method was investigated to return the ferroresonance stress to its stabilized state (Heidary \& Radmanesh, 2018). Y1ldirım et al investigated the frequency properties of disturbances in the voltage waveform caused by the ferroresonance phenomenon by Fourier transform (Yildirim et al., 2009). Akinrinde et al. examined the effects of ferroresonance phenomena on the characteristics of the wind turbine-driven generator (Akinrinde et al., 2019). Bo and Tiecheng analyzed the disturbances in the grid voltage wave subjected to the ferroresonance phenomena to assist in the detection of ferroresonance phenomena with wavelet transform (Zhang \& Lu, 2009).

The ferroresonance phenomenon is often confused with transformer switching, capacitor 
switching, and load switching processes. Since a simple switching operation is very similar to ferroresonance, studies that distinguish these events from each other are quite common in the literature. In their work Mokryani and Haghifam, they used a multilayer perceptron for ferroresonance detection. They achieved $93 \%$ accuracy in the triple classification they made for capacitor switching, transformer switching, and load switching (Mokryani \& Haghifam, 2008). In another study, artificial neural networks were used to classify overvoltage fluctuations in power transformers during ferroresonance. In the study where different load scenarios were taken into consideration, ferroresonance detection was performed with a rate of $98.75 \%$ (ElNozahy et al., 2012). In a classification study to distinguish between capacitor switching and ferroresonance events, Sharbin et al. proposed a prediction model that recognizes $97 \%$ ferroresonance event with ANN-based wavelet transform (Sharbain et al., 2017). In a study conducted with the same purpose, the adequacy of using wavelet transform and ANN together for determining ferroresonance was examined and investigated (Mokryani et al., 2007). In another study where ANN and wavelet transform were used together, the ferroresonance was detected at a rate of approximately 94\% (Mokryani et al., 2009). Ferroresonance classification, made with a smart tracking and suppression system based on a fuzzy logic algorithm, was made by considering 8 different events and $100 \%$ accuracy was achieved (Wang et al., 2011). Mokryani et al. determined ferroresonance with an accuracy of $97.5 \%$ using the combination of support vector machines (SVM) and S-transform (ST) (Mokryani et al., 2010). Uzunoğlu and Uğur proposed Kalman filters for analysis and detection of voltage wavelength and amplitude during the chaotic ferroresonance phenomenon (Uzunoglu \& Ugur, 2013). In a study carried out to ensure that ferroresonance is extracted from the aforementioned phenomena and detected, it has been suggested that the vibration difference of the voltage transformer can be utilized. It is thought that differentiating the vibration exhibited by a transformer during ferroresonance and the vibration caused by the switching of any equipment can be used in determining ferroresonance (Arroyo et al., 2019). In a classification and recognition study based 
on the sparse autoencoder (SAE), ferroresonance was detected at 97\% (Chen et al., 2018). In a study conducted to understand the causes of ferroresonance and to take precautions, ferroresonance analysis and determination were carried out utilizing an algorithm. For this purpose, first of all, some parameters in the power system were thought to trigger ferroresonance, and these parameters were eliminated with the iterative elimination algorithm, and analysis was performed (Yang et al., 2018).

After examining the previous studies, it is understood that the ferroresonance phenomenon has not been solved in electrical power systems yet, and sufficient precautions cannot be taken and more scientific studies are needed on this subject. As far as we have examined, we have yet to come across studies that can predict the voltage waveform and its behavior in the first second of the ferroresonance phenomenon, especially in voltage distortions during ferroresonance. It is the main purpose and motivation source of this study to eliminate this gap to some extent and to present a model that can predict the voltage distortions and amplitudes of the ferroresonance phenomenon at the very beginning of the first second. To achieve this goal, multi-step LSTM cells connected with an algorithm were designed in this study, and a combined LSTM network was constructed and implemented that predicts the future data with very little input. The rest of the study is organized as follows. In the second section, basic information about the method used in the study is given. The proposed combined LSTM network is introduced and explained in the third section. In the fourth section, obtaining the data set and experimental results are explained. The fifth section includes evaluation of results and discussion. In the sixth section, the conclusion of the study is presented.

\section{PROPOSED METHOD}

In this section, we describe our LSTM-based deep learning prediction framework for distortion in grid phase voltage during the ferroresonance phenomenon in the power system. It is motivated by the combination of eleven LSTM networks, which takes into account the distorted signal of the monophase voltage arising during the time interval of the ferroresonance 
phenomenon.

\section{Proposed Framework Overview}

The internal structure of the proposed deep neural network is shown in Figure 4 and its algorithm is shown in Figure 5. The proposed deep neural network model was created by consecutively connecting 11 LSTM cells. In this combined LSTM model, the inputs are the information of the values between the first 0 and 751th samples of the point voltage samples taken during the ferroresonance occurring in the electric power network, and the outputs represents the forecasting of the samples from 751 to 17738.

Unlike ordinary LSTM models, since the ferroresonance moment is only a few seconds, sampling is used instead of time on the horizontal axis. In Figure 4, T1 represents both the training data of the whole combined LSTM structure and the training data of the LSTM1. T1 also represents the input of the proposed combined LSTM model. In Figure 4, all P values from P1 to P11 represent the output of all modules from LSTM1 to LSTM11 respectively, as well as the output values obtained as a result of the test process applied to these modules. As can be seen from the algorithm in Figure 5, each LSTM module from LSTM1 to LSTM11 is trained with $75 \%$ of the data allocated for them and tested with $25 \%$. For the combined LSTM model obtained through the algorithm, only about $5 \%$ of the data is training data, the remaining data is test data or output of the system. The final forecasting is generated after a fully-connected layer in the last LSTM module LSTM11. Each of the LSTM modules used in the proposed method is used to achieve long-term dependency. Since each LSTM module is connected consecutively, the inputs for each LSTM structure have been reshaped as shown in the algorithm in Figure 5 and in the internal structure of the combined model in Figure 4. Figure 3 below shows the common block diagram used separately for each of the LSTM modules connected in the proposed combined model. The final forecasting is generated after a fullyconnected layer in the last module (LSTM11). 


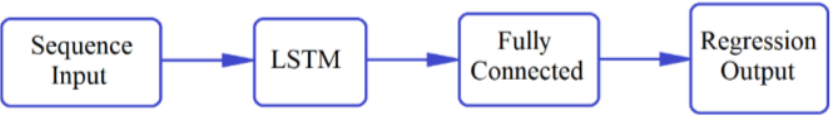

Figure 3 Common block diagram for an LSTM module

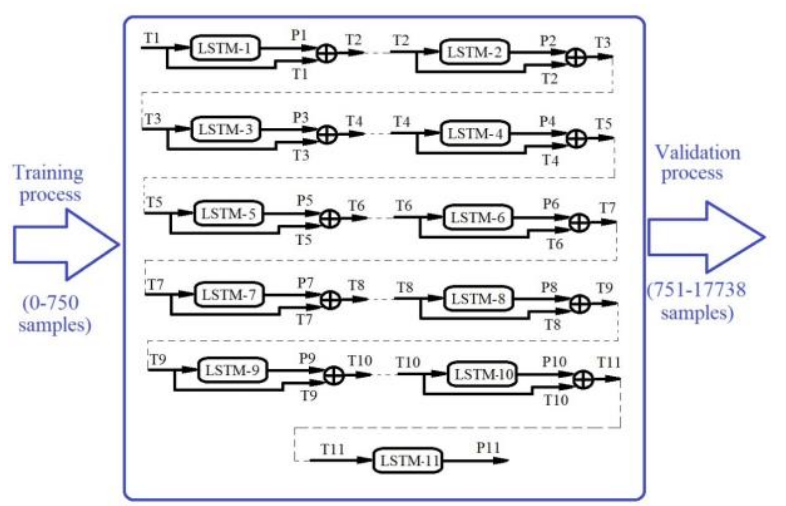

Figure 4 Inner structure of proposed combined LSTM

\section{Model Assessment Indexes}

Mean Absolute Error (MAE), Mean Absolute Percent Error (MAPE) and Root Mean Square Error (RMSE) are used to evaluate the performance of the proposed model. Error measurements are expressed as follows (Manowska, A. 2020 \& Liu et al., 2018):

$$
\begin{aligned}
& M A E=\frac{1}{n} \sum_{i=1}^{n}\left|\left(\hat{y}_{i}-y_{i}\right)\right| \\
& M A P E \frac{1}{n} \sum_{i=1}^{n}\left|\left(\frac{\hat{y}_{i}-y_{i}}{y_{i}}\right)\right| \\
& R M S E=\sqrt{\frac{1}{n} \sum_{i=1}^{n}\left(\hat{y}_{i}-y_{i}\right)^{2}}
\end{aligned}
$$

where $\mathrm{n}$ is the size of the training or test samples and $\hat{y} \mathrm{i}$ and yi are the predicted value and the actual value, respectively. MAE is the mean of absolute errors between forecasting values and actual values. MAPE is the ratio between the error and the actual value. The RMSE gives the sample standard deviation of the differences between predicted values and actual observed values. The lower the MAE, MAPE, and RMSE values, the better the prediction performance (Liu et al., 2018). 


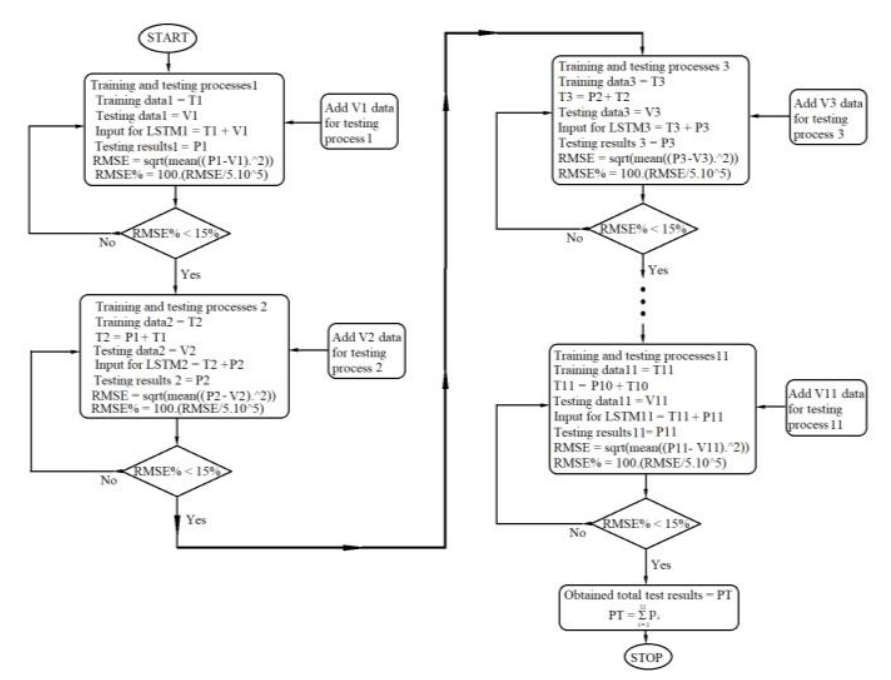

Figure 5 Operation of the proposed combined LSTM

\section{EXPERIMENTS AND RESULTS}

The proposed model was applied to predict the voltage distortion during a real-world ferroresonance event. In this section, experiments are explained in detail and the results obtained from the proposed combined LSTM model are presented.

\section{Definition of Data Set}

In this study, the data set is obtained by using one-to-one modeling of an electricity network. As shown in Figure 6, the 380 kV Electric Power System network Seyitömer-Isıklar in Turkey modeling it was performed using the actual parameters in MATLAB Simulink environment.

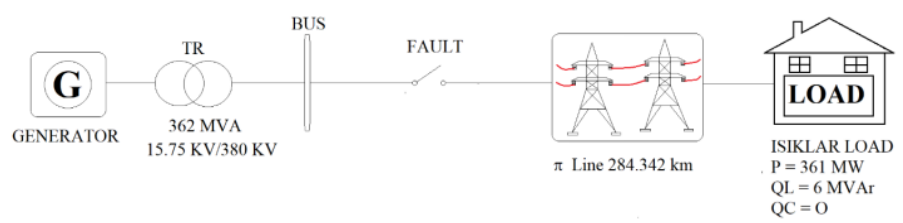

Figure 6 Seyitomer-Isıklar electric power network

In Figure 7, the MATLAB Simulink model of the Seyitomer-Isıklar electrical power distribution network built according to the real parameters in Table 1 is shown. A realistic ferroresonance phenomenon can be generated by sudden power cuts. 
With the Simulink model, the expansion of the voltage and the ferroresonance were achieved by using breakers to create a realistic ferroresonance phenomenon.

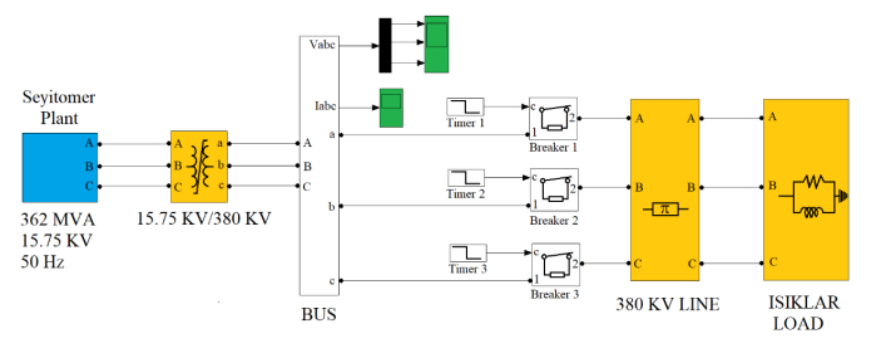

Figure 7 Seyitomer-Isıklar Matlab-Simulink model

In the modeling, the required high voltage and the load fed by the medium transmission line are used to represent a realistic ferroresonance phenomenon. The graph of the phase $\mathrm{R}$ measured in the network, which is exposed to the ferroresonance phenomenon suddenly while working stably, is shown in Figure 8 . The graph in Figure 7 is obtained for a 4.5 -second interval before and after ferroresonance.

Table 1 Parameters in Seyitomer-Is1klar power network

\begin{tabular}{|c|c|}
\hline Components & Parameters \\
\hline Transformers & $362 \mathrm{MVA}, 1.75 \mathrm{kV} / 380 \mathrm{kV}$ \\
\hline Generators & $362 \mathrm{MVA}, 1.75 \mathrm{kV}, 50 \mathrm{~Hz}$ \\
\hline Line Length & $284.341 \mathrm{~km}$ \\
\hline Load & $361 \mathrm{MW}, 6 \mathrm{MVAr}$ \\
\hline
\end{tabular}

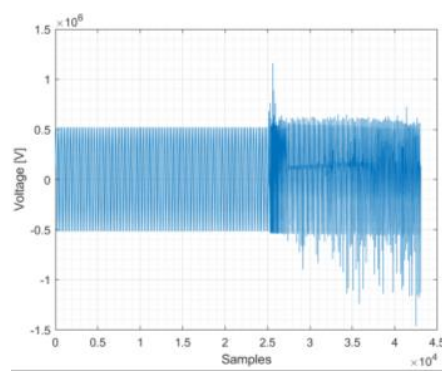

(a)

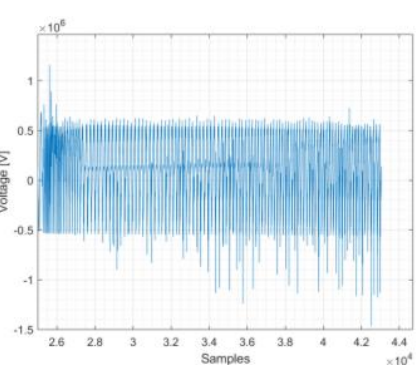

(b)

Figure 8 Voltage variation for R phase (a) whole wave form, (b) ferrorezonance phenomena

Figure 8 (b) shows the graph of the R phase voltage used in our study during the ferroresonance phenomenon. Using this graph, the sample numbers in the training and test data set shown in Table 2 were determined for each LSTM module in accordance with the algorithm in Figure 
5. The sample numbers and input data voltage graphs determined for each module in Table 2 are given in Figure 9.

Table 2 Training and testing data summary for all LSTM modules

\begin{tabular}{|l|l|c|c|c|c|}
\hline \multirow{2}{*}{ Modules } & \multicolumn{2}{|c|}{ Training data } & \multicolumn{2}{c|}{ Testing data } & \multirow{2}{*}{ Total } \\
\cline { 2 - 5 } & Number & Nomenclature & Number & Nomenclature & \\
\hline LSTM1 & 750 & $\mathrm{~T} 1$ & 250 & $\mathrm{~V} 1$ & 1000 \\
\hline LSTM2 & 1000 & $\mathrm{~T} 2$ & 333 & $\mathrm{~V} 2$ & 1333 \\
\hline LSTM3 & 1333 & $\mathrm{~T} 3$ & 444 & $\mathrm{~V} 3$ & 1777 \\
\hline LSTM4 & 1777 & $\mathrm{~T} 4$ & 592 & $\mathrm{~V} 4$ & 2369 \\
\hline LSTM5 & 2369 & $\mathrm{~T} 5$ & 789 & $\mathrm{~V} 5$ & 3158 \\
\hline LSTM6 & 3158 & $\mathrm{~T} 6$ & 1052 & $\mathrm{~V} 6$ & 4210 \\
\hline LSTM7 & 4210 & $\mathrm{~T} 7$ & 1403 & $\mathrm{~V} 7$ & 5613 \\
\hline LSTM8 & 5613 & $\mathrm{~T} 8$ & 1871 & $\mathrm{~V} 8$ & 7484 \\
\hline LSTM9 & 7484 & $\mathrm{~T} 9$ & 2494 & $\mathrm{~V} 9$ & 9978 \\
\hline LSTM10 & 9978 & $\mathrm{~T} 10$ & 3326 & $\mathrm{~V} 10$ & 13304 \\
\hline LSTM11 & 13304 & $\mathrm{~T} 11$ & 4434 & $\mathrm{~V} 11$ & 17738 \\
\hline Combined & 750 & $\mathrm{~T} 12$ & 16988 & $\mathrm{~V} 12$ & 17738 \\
\hline
\end{tabular}

\section{Experimental Results and Analysis}

In power systems, the ferroresonance phenomenon, which causes distortions in phase voltage and subsequent damage to many equipment, can start and end in a very short time such as 5 seconds. Therefore, as soon as the ferroresonance phenomenon begins, it is an important requirement to predict the behavior of the grid phase voltage during the phenomenon and take precautions in a very short time. In this study, an combined LSTM model was designed and applied by using 17,438 samples of the $\mathrm{R}$ phase voltage during the ferroresonance phenomenon. Using only the first 750 of these samples the remaining samples (instantaneous voltage values) were estimated. In other words, the training was performed with approximately $5 \%$ of all data and the test was performed with the remaining approximately $95 \%$ data, unlike normal. The remaining 16,988 data were estimated by taking 750 data samples. For this purpose, 11 LSTM modules are connected in a chain. Output values forecasted with each module are added as an input to the next module. Test results obtained from each module are given in Figure 10 with error graphs. The success and performance of each model according 
to the MAE, MAPE, and RMSE values, which we accept as the evaluation index, can be observed in Table 3.

Table 3 Experimental results in terms of evaluation indexes

\begin{tabular}{|l|l|l|l|}
\hline Modules & RMSE $\left.(\mathbf{x ~ 1 0})^{4}\right)$ & MAPE & MAE $(\mathbf{x ~ 1 0})^{3}$ \\
\hline LSTM1 & 5.8279 & 0.0117 & 1.1804 \\
\hline LSTM2 & 6.1444 & 0.0063 & 11.719 \\
\hline LSTM3 & 4.2043 & 0.0162 & 3.9655 \\
\hline LSTM4 & 5.2282 & 0.0387 & 4.2162 \\
\hline LSTM5 & 4.9726 & 0.0287 & 2.2312 \\
\hline LSTM6 & 6.8244 & 0.0441 & 1.7191 \\
\hline LSTM7 & 3.7345 & 0.0118 & 3.6269 \\
\hline LSTM8 & 4.8548 & 0.0203 & 4.2413 \\
\hline LSTM9 & 7.9320 & 0.0406 & 0.6960 \\
\hline LSTM10 & 8.4313 & 0.0359 & 6.7913 \\
\hline LSTM11 & 8.5389 & 0.0484 & 2.8575 \\
\hline Combined LSTM & 7.2411 & 0.0346 & 1.8723 \\
\hline
\end{tabular}

The error and estimation graphs obtained at the output of each model are presented separately in Figure 10. The successful result of the study depends on the success of each module. As shown in Table 3, as a result of the successful performance of each module, the results produced by the combined LSTM model are also given in the bottom column of Table 3 . Unlike normal LSTM models, with a new strategy, using only 750 of 17738 data as training data, 16988 voltage values were successfully estimated as shown in Figure 11. MAE and MAPE values were added to the algorithm later. According to Table 2, the MAE value in LSTM2 is seen as the highest due to the high voltage points reaching their maximum level, while the MAPE value is also the lowest. As the number of modules progresses from 1 to 11 , the number of data used increases. Therefore, quite different performance values can be obtained in each module. In this sense, the error values are different from each other, although they are all within the desired limits. RMSE values range from the lowest $3.7 \times 104$ to the highest $8.5 \times 104$, with no apparent deviation. Considering the MAPE values, it ranges between the lowest 0.006 and the highest 0.05 . Similarly, there was no significant difference in MAPE values, and results supporting the operation of the combined system were obtained. 


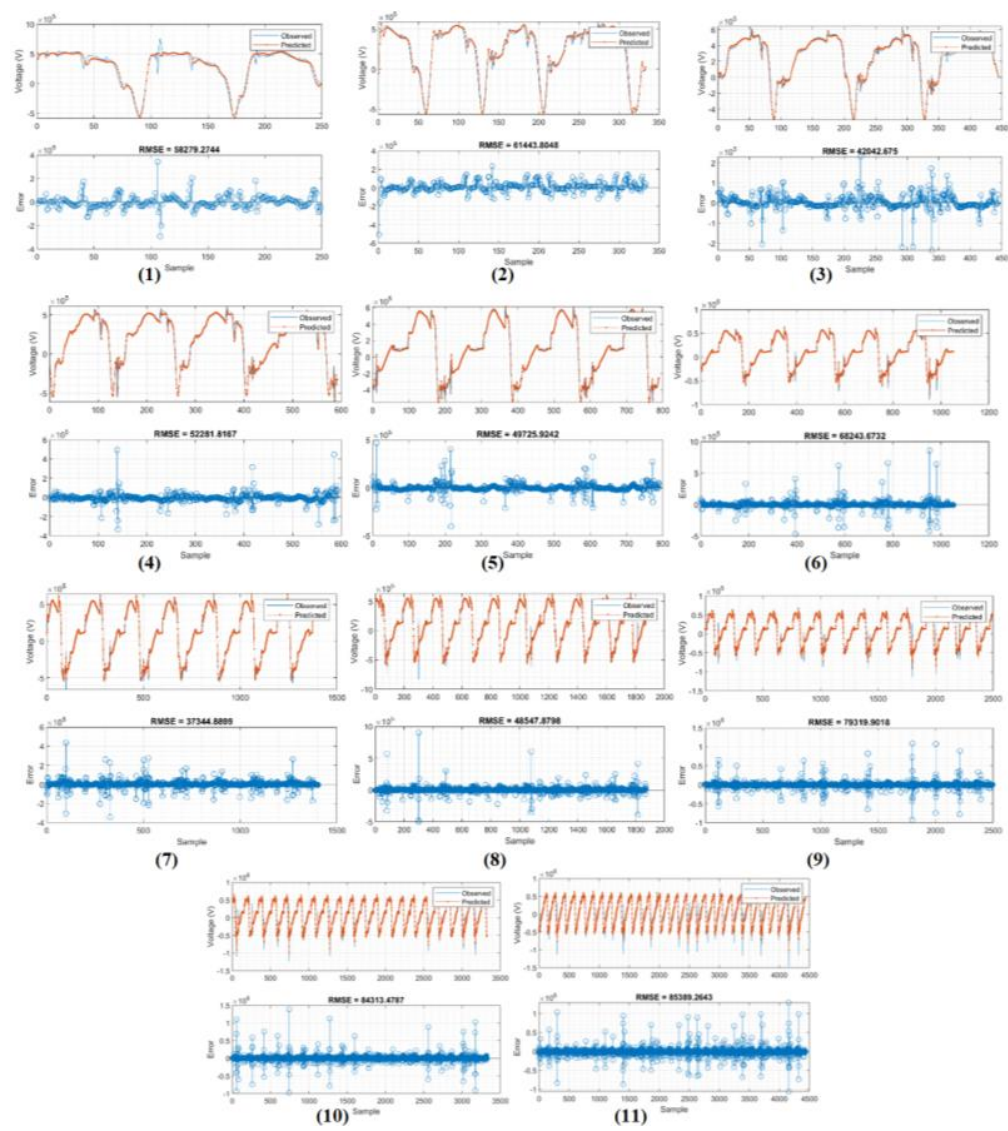

Figure 10 Testing results and errors; (1): results of LSTM1; (2): results of LSTM2, (3): results of LSTM3,; (4): results of LSTM4 ; (5): results of LSTM5 ; (6): results of LSTM6; (7): results of LSTM7; (8): results of LSTM8; (9): results of LSTM9; (10): results of LSTM10; (11): results of LSTM11.

The MAE value is also more successful than some LSTM modules as $1.8 \times 103$ supporting other evaluation criteria. The results obtained from the sub LSTM modules also confirm the results obtained from the combined model. These new results achieved by LSTM (combined) with the new strategy are extremely important and original, considering the test and training rates (approximately 5\% training data and 95\% test data).

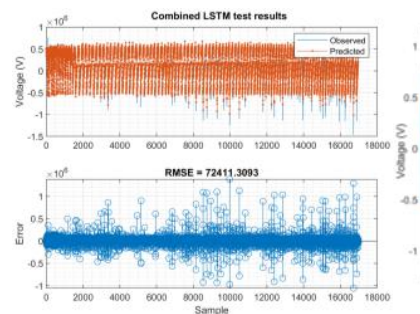

(a)

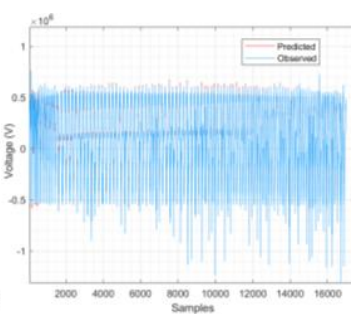

(b)

Figure 11 Resultss for combined LSTM (a) testing and error (b) forecasting results 


\section{DISCUSSION}

Each of the 11 LSTM modules used in the combined LSTM model applied in the study was trained with $75 \%$ of the data allocated for it and tested with $25 \%$ test data included later. Results from these LSTM modules confirm the results from the combined LSTM model. A disadvantage in the combined LSTM model is that test data for each LSTM module is entered externally. This can also lead to the difficulty of finding suitable test data. Detecting the ferroresonance phenomenon in the first moments of the first second and determining the voltage behavior is a great advantage for power systems. The reliability of the study can be proven in two ways. First, the results of the 11 LSTM modules used in the combined LSTM model and the results of the combined LSTM model support each other. This situation proves the accuracy and reliability of the study. Second, all LSTM modules are combined using the backpropagation learning algorithm shown in figure 5. In this algorithm, if the RMSE ratio is above the desired level, the model is tested again. Therefore, the test can sometimes be repeated over and over again. As a result of this process, the test result may never be at the desired level and the system can continue forever in a continuous circle. The operation of the system and the desired result and reaching the last step are also a guarantee of accuracy and reliability for this model. On the other hand, this situation can be shown as another disadvantage of this system. In the future, this algorithm can be further developed, and advanced combined-hybrid systems combined with convolutional neural network (CNN) can be developed and implemented.

\section{CONCLUSION}

This study presents a multi-step deep learning framework that can predict changes in the behavior of phase voltage during ferroresonance phenomena in power systems from the very beginning. This deep learning framework is based on the principle of using LSTM modules in a combined way by connecting them through an algorithm. Each LSTM module used in the 
study was trained with $75 \%$ of the data set and tested with $25 \%$. If the test results obtained from each LSTM module are at the desired level, they are added to the input of the next LSTM module. The test results from each LSTM module support the results obtained from the combined model presented. The combined model was trained with only 750 samples of the whole data set consisting of 17738 samples and was tested with a data set with a much larger number of samples, 16988. Although the rate of the test sample is much higher than that of the training sample, both the successful application of the combined system and the presence of the backpropagation learning algorithm resulted in extremely successful estimation results presented in Figure 10 and Figure 11.

\section{Acknowledgment}

This study was funded by the Scientific Research Projects Coordination Unit of Kayseri University. Project ID: 1025

\section{REFERENCES}

Akinrinde, A.O., Swanson, A.G., \& Tiako, R. 2019. Dynamic Behavior of Wind Turbine Generator Configurations during Ferroresonant Conditions. Energies, 12, 639.

Arroyo, A., Martinez, R., Manana, M., Pigazo, A., \& Mínguez, R. 2019. Detection of ferroresonance occurrence in inductive voltage transformers through vibration analysis. International Journal of Electrical Power \& Energy Systems, 106, 294-300.

Boutora, S., \& Bentarzi, H. 2018. Study of ferroresonance favorable cases in electric substations, Rev. Roum. Sci. Techn.- Électrotechn. et Énerg. 63, 4, 359-364.

Chen, K., Hu, J., \& He, J. 2018. A Framework for Automatically Extracting Overvoltage Features Based on Sparse Autoencoder. IEEE Transactions on Smart Grid, 9, 594-604.

EINozahy, M., El-Shatshat, R.A., \& Salama, M. 2012. A robust technique for overvoltages classification in power transformers. 2012 IEEE Power and Energy Society General Meeting, 1-8. 
Heidary, A., \& Radmanesh, H. 2018. Smart solid-state ferroresonance limiter for voltage transformers application: principle and test results. Iet Power Electronics, 11, 2545-2552.

Liu, H., Xiwei, M., \& Li, Y. 2018. Wind speed forecasting method based on deep learning strategy using empirical wavelet transform, long short term memory neural network and Elman neural network. Energy Conversion and Management, 156, 498-514.

Li, J. 2017. The 10 Deep Learning Methods AI Practitioners Need to Apply. Available online: https: //medium.com/cracking-the-data-science-interview/the-10-deep-learning-methods-aipractitioners-needto-apply-885259f402c1 (accessed on Dec 24 2020).

Manowska, A. 2020. Using the LSTM Network to Forecast the Demand for Electricity in Poland. Applied Sciences, 10, 8455.

Mokryani, G., \& Haghifam, M. 2008. Application of wavelet transform and MLP neural network for Ferroresonance identification. 2008 IEEE Power and Energy Society General Meeting - Conversion and Delivery of Electrical Energy in the 21st Century, 1-6.

Mokryani, G., Haghifam, M.R., \& Esmaeilpoor, J. 2007. Identification of ferroresonance based on wavelet transform and artificial neural networks. 2007 Australasian Universities Power Engineering Conference, 1-6.

Mokryani, G., Haghifam, M., Latafat, H., Aliparast, P., \& Abdolahi, A. 2009. Wavelet Based Kernel Fisher Classifier For Ferroresonance Identification. 2009 15th International Conference on Intelligent System Applications to Power Systems, 1-6.

Mokryani, G., Siano, P., \& Piccolo, A. 2010. Identification of ferroresonance based on Stransform and support vector machine. Simul. Model. Pract. Theory, 18, 1412-1424.

Rezaei, S. 2017. Adaptive overcurrent protection against ferroresonance. Iet Generation Transmission \& Distribution, 12, 1573-1588.

Sharbain, H.A., Osman, A., \& El-Hag, A. 2017. Detection and identification of ferroresonance. 2017 7th International Conference on Modeling, Simulation, and Applied Optimization (ICMSAO), 1-4. 
Tugai, I. 2015. Investigation of ferroresonance in electrical networks at open-phase operating conditions. Computational Problems of Electrical Engineering, 5, 1.

Uzunoglu, C.P., \& Ugur, M. 2013. Adaptive detection of chaotic oscillations in ferroresonance using modified extended Kalman filter. Turkish Journal of Electrical Engineering and Computer Sciences, 21, 1871-1879.

Wang, J., Yang, Q., Sima, W., Yuan, T., \& Zahn, M. 2011. A Smart Online Over-Voltage Monitoring and Identification System. Energies, 4, 599-615, doi:10.3390/en4040599

Yang, M., Sima, W., Chen, L., Duan, P., Sun, P., \& Yuan, T. 2018. Suppressing ferroresonance in potential transformers using a model-free active-resistance controller. International Journal of Electrical Power \& Energy Systems, 95, 384-393.

Yang, M., Sima, W., Duan, P., Zou, M., Peng, D., Yang, Q., \& Duan, Q. 2017a. Electromagnetic transient study on flexible control processes of ferroresonance. International Journal of Electrical Power \& Energy Systems, 93, 194-203.

Yildirim, S., Akinci, T.C., Seker, S., \& Ekren, N. 2009. Determination of the Characteristics for Ferroresonance Phenomenon in Electric Power Systems. World Academy of Science, Engineering and Technology, International Journal of Electrical, Computer, Energetic, Electronic and Communication Engineering, 3, 1422-1426.

Zhang, B., \& Lu, T. 2009. On the Use of Wavelet Decomposition for Ferroresonance Detection in Power System. 2009 Asia-Pacific Power and Energy Engineering Conference, 1-4. 